Article

\title{
Experimental and Theoretical Study of an Actively Q-Switched Tm:YLF Laser with an Acousto-Optic Modulator
}

\author{
Lei Guo ${ }^{1}$, Yaling Yang ${ }^{1}$, Ruihua Wang ${ }^{2}$, Baitao Zhang ${ }^{2}$, Tao Li ${ }^{3,4,5}$, Shengzhi Zhao ${ }^{3}$, Jingliang He ${ }^{2}$ \\ and Kejian Yang $2,3,4,5,6, *$
}

1 School of Mechanics and Photoelectric Physics, Anhui University of Science and Technology, Huainan 232001, China; leiguo@aust.edu.cn (L.G.); yalingyang_opt@163.com (Y.Y.)

2 Institute of Novel Semiconductors, State Key Laboratory of Crystal Materials, Shandong University, Jinan 250100, China; rhwang@sdu.edu.cn (R.W.); btzhang@sdu.edu.cn (B.Z.); jlhe@sdu.edu.cn (J.H.)

3 China Key Laboratory of Laser \& Infrared System (Ministry of Education), Shandong Provincial Key Laboratory of Laser Technology and Application, School of Information Science and Engineering, Shandong University, Qingdao 266237, China; litao@sdu.edu.cn (T.L.); shengzhi_zhao@sdu.edu.cn (S.Z.)

4 Collaborative Innovation Center of Light Manipulations and Applications, Shandong Normal University, Jinan 250358, China

5 Shenzhen Research Institute of Shandong University, Shenzhen 518057, China

6 State Key Laboratory of Quantum Optics and Quantum Optics Devices, Shanxi University, Taiyuan 030006, China

* Correspondence: k.j.yang@sdu.edu.cn

Citation: Guo, L.; Yang, Y.; Wang, R.; Zhang, B.; Li, T.; Zhao, S.; He, J.; Yang, K. Experimental and Theoretical Study of an Actively Q-Switched Tm:YLF Laser with an Acousto-Optic Modulator. Molecules 2021, 26, 7324. https://doi.org/10.3390/ molecules26237324

Academic Editor: Elena Cariati

Received: 13 November 2021

Accepted: 1 December 2021

Published: 2 December 2021

Publisher's Note: MDPI stays neutral with regard to jurisdictional claims in published maps and institutional affiliations.

Copyright: (c) 2021 by the authors. Licensee MDPI, Basel, Switzerland. This article is an open access article distributed under the terms and conditions of the Creative Commons Attribution (CC BY) license (https:/ / creativecommons.org/licenses/by/ $4.0 /)$.
Abstract: We report the characteristics of a diode-end-pumped, high-repetition-rate, acoustic-optic (AO) Q-switched Tm:YLF laser operating from $5 \mathrm{kHz}$ to $10 \mathrm{kHz}$. In the continuous-wave (CW) regime, a maximum average output power of $8.5 \mathrm{~W}$ was obtained with a slope efficiency of $30.7 \%$. Under the AO Q-switching regime, a maximum output power of $7.32 \mathrm{~W}$ was obtained at a repetition frequency of $5 \mathrm{kHz}$ with a pulse width of $68 \mathrm{~ns}$ and a pulse energy of $1.4 \mathrm{~mJ}$, corresponding to a peak power of $21.5 \mathrm{~kW}$. A time-dependent rate equation model is introduced to theoretically analyze the results obtained in the experiment, in which the cross-relaxation phenomenon, upconversion losses and ground-state depletion are taken into account. Additionally, the evolution processes of population inversion density and intracavity photon number density with time are also presented. The theoretical results well predict the dependence of laser output characteristics of Tm:YLF crystal on the incident pump powers.

Keywords: actively Q-switched; Tm:YLF laser; rate equation model

\section{Introduction}

Recently, 2-micrometer lasers have attracted much attention in the fields of laser medicine, material processing, laser communication, atmospheric pollution monitoring and mid-infrared optical parametric oscillator. In particular, the high-repetition-rate and short-pulse-width laser source plays an increasingly important role in these fields. As is known to all, the trivalent rare earth ion thulium $\left(\mathrm{Tm}^{3+}\right)$ is a common active ion emitting at $2 \mu \mathrm{m}$ with the advantages of long fluorescence lifetime and high quantum efficiency, and its pump source is a commercially available laser diode (LD) [1]. Up to now, various $\mathrm{Tm}^{3+}$ ion-doped crystals (YAP, YAG, LuAG, YLF, etc.) have been widely investigated to achieve a 2-micrometer pulsed laser based on the Q-switching technique [2-4]. As a typical representative, Tm:YLF, a natural birefringent crystal with a linearly polarized output, has been used to achieve as high as $87.5 \mathrm{~W}$ of CW laser output power at $1907.8 \mathrm{~nm}$ [5]. Under the Q-switching regime, Jabczyński et al. reported an acoustic-optic (AO) Q-switched Tm:YLF laser with a pulse width of $20 \mathrm{~ns}$ and a pulse energy of $10.5 \mathrm{~mJ}$ at a repetition frequency of $10 \mathrm{~Hz}$, corresponding to the peak power of nearly $0.5 \mathrm{MW}$ [6]. In 2015, Korenfeld et al. demonstrated a passively Q-switched diode-pumped Tm:YLF laser based 
on a Cr:ZnSe saturable absorber with a maximum pulse energy of $4.22 \mathrm{~mJ}$ and a pulse duration of $26 \mathrm{~ns}$ at the repetition rate of $400 \mathrm{~Hz}$, corresponding to the peak power of $162.3 \mathrm{~kW}$ [7]. In 2018, Sheintop et al. demonstrated a high-energy and narrow-bandwidth tunable Tm:YLF laser using a pair of Etalon plates. At a repetition rate of $1 \mathrm{kHz}$, a pulse energy of $1.97 \mathrm{~mJ}$ and a pulse duration of $37 \mathrm{~ns}$ were achieved at $1879 \mathrm{~nm}$ with a full width at half-maximum (FWHM) of $0.15 \mathrm{~nm}$, corresponding to a peak power of $53.2 \mathrm{~kW}$ [8]. However, these studies focus on high-energy lasers and not the research of high-power and high-repetition-rate laser sources.

On the other hand, with the development of the energy level transition mechanism of the $\mathrm{Tm}^{3+}$-doped quasi-three-level laser, the rate equation model has been established to analyze laser operating characteristics theoretically, which reveals a good agreement with the experimental results under the $\mathrm{CW}$ and passively Q-switched regimes $[9,10]$. However, there are few numerical simulation studies on actively Q-switched Tm lasers. In the existing models, the laser performances are theoretically investigated by solving the steady-state rate equations to reduce computational complexity, which makes it difficult to simulate the time evolution of photon number and population inversion. Additionally, some key details cannot be presented, such as the pulse build-up time and the evolution of pulse at the initial stage.

In this paper, we demonstrated a high-repetition-rate AO Q-switched Tm:YLF laser. Under CW operation, a maximum average output power of $8.5 \mathrm{~W}$ was obtained with a slope efficiency of $30.7 \%$. In the Q-switching regime, a maximum output power of $7.32 \mathrm{~W}$ was realized with the shortest pulse width of $68 \mathrm{~ns}$ and a maximum pulse energy of $1.4 \mathrm{~mJ}$ under a repetition rate of $5 \mathrm{kHz}$, corresponding to a peak power of $21.5 \mathrm{~kW}$. Besides, we also built a rate equation theoretical model of the diode-end-pumped Tm:YLF laser to theoretically analyze the results obtained in the experiment.

\section{Experimental Setup}

Figure 1 shows the experimental setup of the diode-end-pumped AO Q-switched Tm:YLF laser. The a-cut Tm:YLF crystal had a doping concentration of 3.5 at. $\%$ with a dimension of $3 \mathrm{~mm} \times 3 \mathrm{~mm} \times 10 \mathrm{~mm}$. The crystal was wrapped in indium foil and mounted in a copper block cooled by water to $18^{\circ} \mathrm{C}$. Both surfaces were antireflection (AR)coated from $750 \mathrm{~nm}$ to $850 \mathrm{~nm}$ and $1800 \mathrm{~nm}$ to $2150 \mathrm{~nm}$. A $35 \mathrm{~W}$ fiber-coupled LD emitting at $790 \mathrm{~nm}$ with a core diameter of $200 \mu \mathrm{m}$ and a numerical aperture of 0.22 was used as the pump source. The pump light was focused into the laser crystal through a 1:2 imaging module with a spot diameter of $400 \mu \mathrm{m}$. The used two-dimensional AO Q-switch (The 26th Electronics Institute of Chinese Ministry of Information Industry) which was made of fused quartz with a physical length of $50 \mathrm{~mm}$ and an acoustic aperture of $3 \mathrm{~mm} \times 3 \mathrm{~mm}$, had a diffraction efficiency of $90 \%$. To reduce the insertion losses, both surfaces of the fused quartz were AR-coated at $2 \mu \mathrm{m}$. The flat-concave cavity was designed with a length of $80 \mathrm{~mm}$. M1 was a flat input mirror and high reflectivity (HR)-coated from $1850 \mathrm{~nm}$ to $2150 \mathrm{~nm}$ (reflectivity $>99.5 \%$ ) and AR-coated from $750 \mathrm{~nm}$ to $850 \mathrm{~nm}$ (reflectivity $<0.5 \%$ ). M2 was a plane-concave output mirror $(R=-200 \mathrm{~mm})$ with the transmission of $20 \%$ from $1850 \mathrm{~nm}$ to $2150 \mathrm{~nm}$. The laser pulse trains were recorded by a fast InGaAs photodetector (EOT, ET-5000) with a rise time of 35 ps and monitored by a digital oscilloscope (1 GHZ bandwidth, Tektronix DPO 7102). The average output power was measured by a laser power meter (PM100, Thorlabs). 


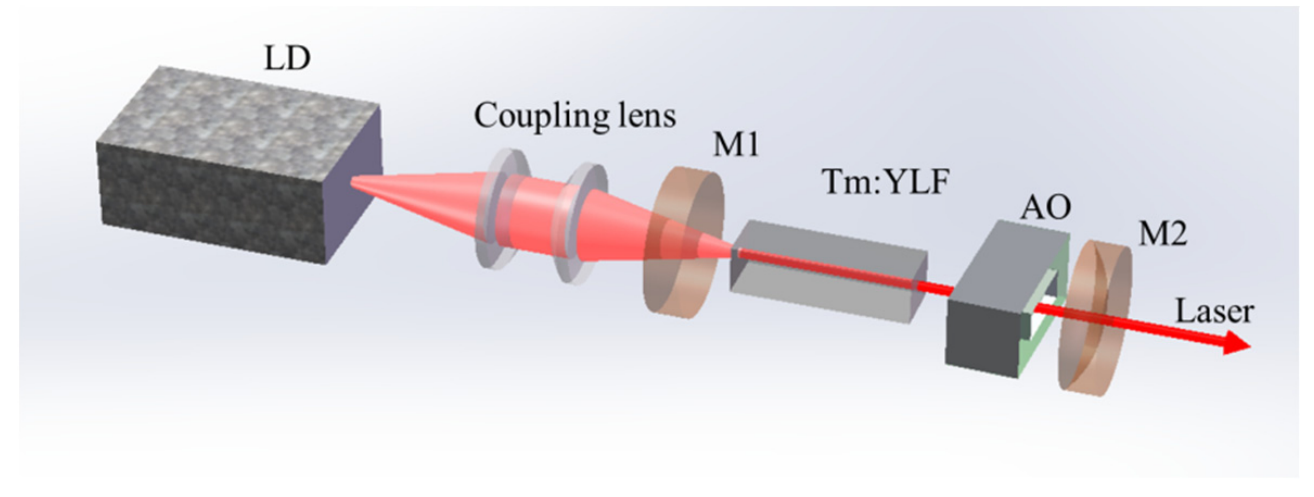

Figure 1. Experimental setup of diode-end-pumped AO Q-switched Tm:YLF laser.

\section{Experimental Results}

Firstly, the CW running performance of the Tm:YLF laser can be seen in Figure 2. Under the pump power of $32 \mathrm{~W}$, the maximum average output power was $8.5 \mathrm{~W}$ for the experiment, corresponding to the slope efficiency of $30.7 \%$.

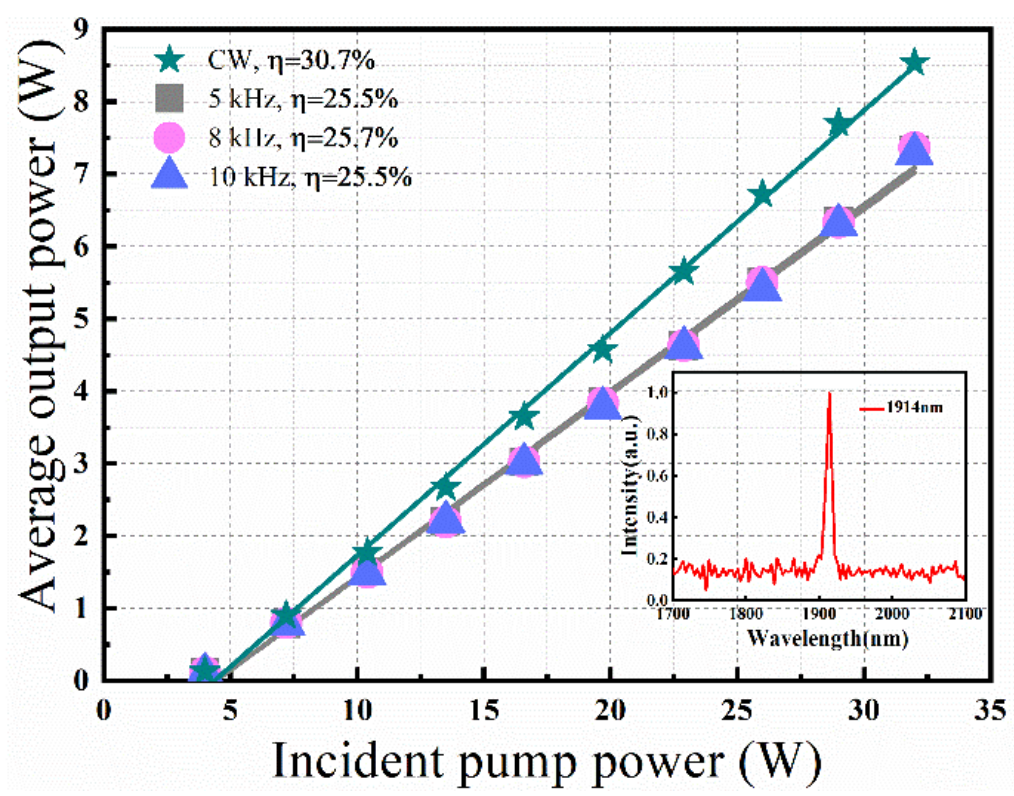

Figure 2. The average output power of diode-pumped CW and AO Q-switched Tm:YLF laser. Inset: output spectrum.

Subsequently, when the AO Q-switch was turned on, stable high-repetition-rate pulse operation was realized with the repetition frequency varying from $5 \mathrm{kHz}$ to $10 \mathrm{kHz}$. As shown in Figure 2, similar output powers and slope efficiencies were obtained under different repetition frequencies. Because when the pulse period is much shorter than the upper-level lifetime of the laser crystal, the spontaneous radiation loss can be ignored. Under the incident pump power of $32 \mathrm{~W}$, the maximum average output powers were $7.32 \mathrm{~W}, 7.36 \mathrm{~W}$ and $7.28 \mathrm{~W}$ under the repetition rates of $5 \mathrm{kHz}, 8 \mathrm{kHz}$ and $10 \mathrm{kHz}$, corresponding to the slope efficiencies of $25.5 \%, 25.7 \%$, and $25.5 \%$. In the inset of Figure 2, the output spectrum was recorded by a spectrometer (AvaSpec-NIR-S-350-2080) at a center wavelength around $1914 \mathrm{~nm}$ with an FWHM of $7 \mathrm{~nm}$. The ONSR (optical signal to noise ratio) for the obtained laser emission was 34.4:1 based on Gaussian fitting. Considering that the resolution of the spectrometer is only $4 \mathrm{~nm}$, the measured spectral width is not accurate.

The dependences of pulse durations, pulse energies and peak powers on incident pump powers at different repetition frequencies are recorded in Figure 3 . When the pump 
power was $32 \mathrm{~W}$, the narrowest pulse durations were $68 \mathrm{~ns}, 114 \mathrm{~ns}$ and $140 \mathrm{~ns}$, and the maximum pulse energies were $1.46 \mathrm{~mJ}, 0.92 \mathrm{~mJ}$ and $0.73 \mathrm{~mJ}$, corresponding to the maximum peak powers of $21.5 \mathrm{~kW}, 8.1 \mathrm{~kW}$ and $5.2 \mathrm{~kW}$ for the repetition frequencies of $5 \mathrm{kHz}, 8 \mathrm{kHz}$ and $10 \mathrm{kHz}$, respectively. However, the repetition frequency would be reduced by half of the set frequency of the AO Q-switch if the incident pump power was lower than $10 \mathrm{~W}$ for frequencies between $8 \mathrm{kHz}$ and $10 \mathrm{kHz}$. The small stimulated emission cross-section of Tm:YLF means that a considerable fraction of Tm ions need be exited during laser operation [11]. Therefore, this phenomenon will occur if the pump intensity is too low or the accumulation time of population inversion is too short, which was also verified in theoretical simulation. Under the maximum pump power, the temporal pulse train at a repetition frequency of $5 \mathrm{kHz}$ is shown in Figure 4 . The bottom of Figure 4 shows the temporal pulse shape with the shortest pulse duration of $68 \mathrm{~ns}$.
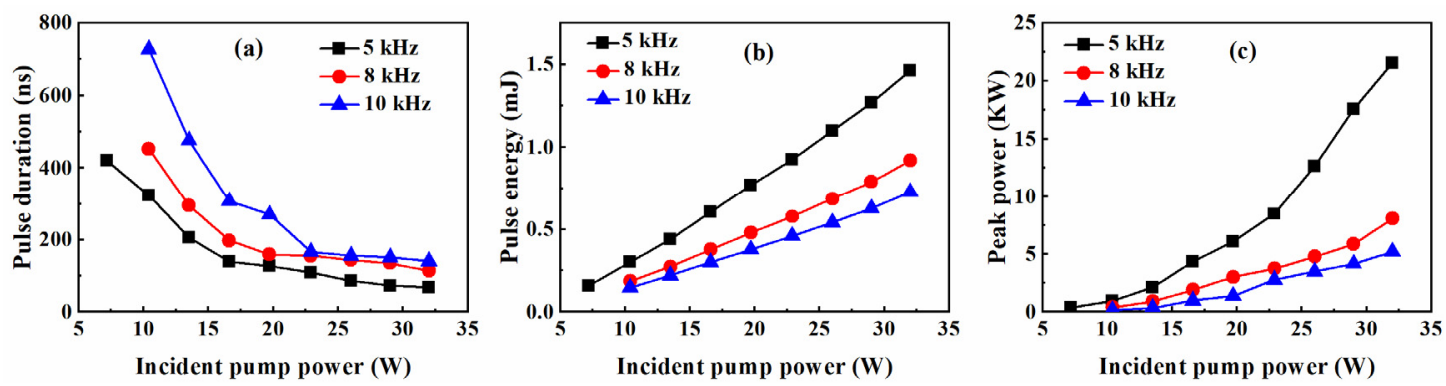

Figure 3. Experiment results for the AO Q-switched Tm:YLF laser: (a) pulse durations, (b) pulse energies and (c) peak powers versus the incident pump powers.

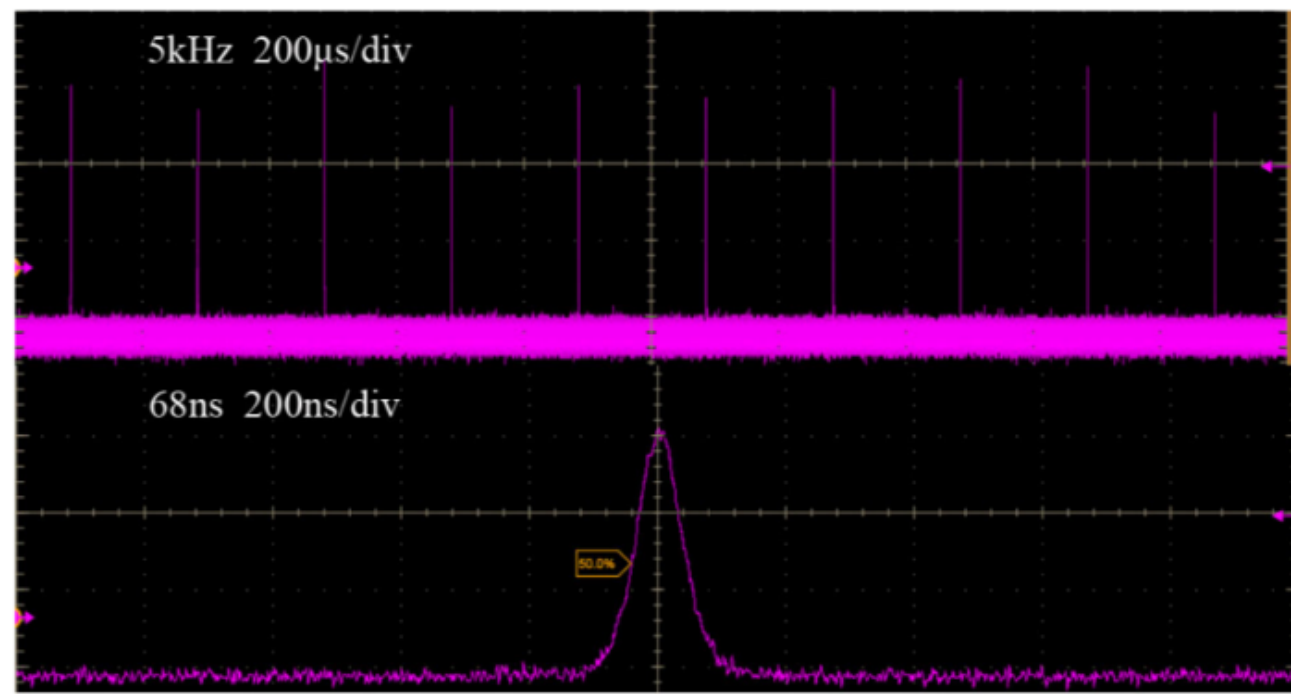

Figure 4. The temporal pulse trains and pulse profile of AO Q-switched Tm:YLF laser at the repetition frequency of $5 \mathrm{kHz}$.

\section{Theoretical Analysis}

To better understand the pulse output characteristics, the quasi-three-level scheme of the $\mathrm{Tm}^{3+}$ ion with energy transfer processes is described in Figure 5. Based on $790 \mathrm{~nm}$ pump sources, the population of the ${ }^{3} \mathrm{H}_{6}$ ground state $\left(\mathrm{N}_{1}\right)$ is excited to the ${ }^{3} \mathrm{H}_{4}$ energy level $\left(\mathrm{N}_{4}\right)$. Due to the short lifetimes of the excited states ${ }^{3} \mathrm{H}_{4}$ and ${ }^{3} \mathrm{H}_{5}\left(\mathrm{~N}_{3}\right)$, the population jumps down to the ${ }^{3} \mathrm{~F}_{4}$ energy level $\left(\mathrm{N}_{2}\right)$ through two non-radiative relaxation (NR) transitions. Finally, the laser emits around $2 \mu \mathrm{m}$ during the transition from ${ }^{3} \mathrm{~F}_{4}$ to ${ }^{3} \mathrm{H}_{6}$. However, unlike the ordinary quasi-three-level structure, there is a strong cross-relaxation (CR) mechanism between the two $\mathrm{Tm}^{3+}$ ions for the ${ }^{3} \mathrm{H}_{4}+{ }^{3} \mathrm{H}_{6} \rightarrow{ }^{3} \mathrm{~F}_{4}+{ }^{3} \mathrm{~F}_{4}$ level transition processes, which results in a high Stokes efficiency of 0.82 , in theory [10]. 


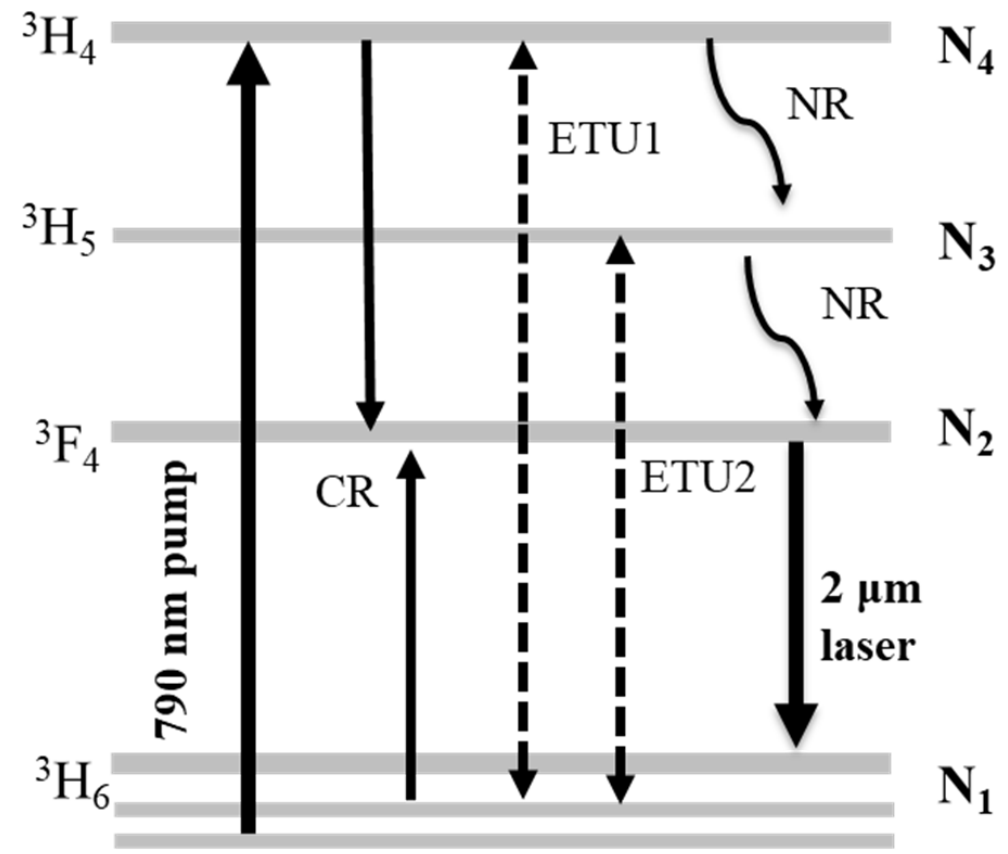

Figure 5. Energy-level transition diagram of $\mathrm{Tm}^{3+}$ ion.

Then, the coupled rate equation model of an actively Q-switched laser, considering the cross-relaxation phenomenon, upconversion losses and ground-state depletion, is introduced to simulate the characteristics of the emitted pulses as follows Equations (1)-(8):

$$
\begin{gathered}
d N_{4} / d t=R_{p}-K_{C R} N_{4} N_{1}+K_{E T U 1} N_{2}{ }^{2}-N_{4} / \tau_{4} \\
d N_{3} / d t=K_{E T U 2} N_{2}{ }^{2}+\beta_{43} N_{4} / \tau_{4}-N_{3} / \tau_{3} \\
d N_{2} / d t=2 K_{C R} N_{4} N_{1}-2\left(K_{E T U 1}+K_{E T U 2}\right) N_{2}{ }^{2}+\beta_{32} N_{3} / \tau_{3}+\beta_{42} N_{4} / \tau_{4}-N_{2} / \tau_{2}-\sigma_{e} c\left(f_{u} N_{2}-f_{l} N_{1}\right) \Phi \\
N_{1}=N_{T m}-N_{2}-N_{3}-N_{4} \\
d \Phi / d t=\sigma_{e} c \Phi\left(f_{u} N_{2}-f_{l} N_{1}\right) l / l^{\prime}-\Phi / \tau_{c} \\
\tau_{c}=t_{r} / \varepsilon \\
t_{r}=2 l^{\prime} / c \\
\varepsilon=-\ln (R)+L+\zeta(t)
\end{gathered}
$$

where $N_{i}(i=1,2,3$ and 4$)$ is the population concentration of the Tm level and $N_{T m}$ is the Tm ion concentration. $K_{C R}$ is the cross-relaxation coefficient. $K_{E T U 1}$ and $K_{E T U 2}$ are the energy transfer upconversion coefficients. The non-radiative lifetime for each level is given by $\tau_{i}$ $(i=2,3,4)$, and the branching ratios for levels $m-n$ are given by $\beta_{n m} . \sigma_{e}$ is the stimulated emission cross-section for the $2 \mu \mathrm{m}$ laser transition. $f_{u}$ and $f_{1}$ are the Boltzmann fractions for the Stark state of ${ }^{3} \mathrm{~F}_{4}$ and ${ }^{3} \mathrm{H}_{6}$ levels. $l^{\prime}=l_{c}+(n-1) l+\left(n_{1}-1\right) l_{1}$ is the optical length of the resonator (here, $l$ and $n$ are the length and the refractive index of the Tm crystal. $l_{1}$ and $n_{1}$ are the length and the refractive index of the AO crystal). $c$ is the speed of light. $\Phi$ is the photon density in the cavity. $\tau_{c}$ is the cavity lifetime. $t_{r}$ is the cavity round-trip transit time. $\varepsilon$ is the cavity round-trip loss. $R$ is the reflectivity of the output mirror. $L$ is the loss in cavity. $\zeta(t)$ is the loss introduced during the operation of Q-switch, which is regarded as a step function $\left(0\right.$ or $\left.\zeta_{\max }\right) . R_{p}$ is the pumping rate and is given by Equation (9):

$$
R_{p}=\eta P_{\text {in }} /\left(\pi r_{p}^{2} l h v_{p}\right)
$$


where $P_{\text {in }}$ is the incident pump power, $r_{p}$ is the pump spot radius, $l$ is the crystal length, $h$ is the Planck's constant and $v_{p}$ is the pump light frequency. Because of the low stimulated emission cross-section of the Tm:YLF crystal, a considerable fraction of the Tm ions need be excited during laser operation. Therefore, considering the ground-state depletion, the absorption coefficient is reduced according to Equation (10):

$$
\eta=\eta_{0} N_{1} / N_{T m}
$$

where $\eta_{0}=80 \%$ is the small-signal pump absorption coefficient. The related parameters are shown in Table 1.

Table 1. Related parameters used in the numerical model [12-16].

\begin{tabular}{cccc}
\hline Parameter & Value & Parameter & Value \\
\hline$h$ & $6.625 \times 10^{-34} \mathrm{~J} \cdot \mathrm{s}$ & $l_{c}$ & $70 \mathrm{~mm}$ \\
$N_{T m}$ & $4.89 \times 10^{20} \mathrm{~cm}^{-3}$ & $\sigma_{e}$ & $3 \times 10^{-21} \mathrm{~cm}^{2}$ \\
$n, n_{1}$ & $1.46,1.44$ & $K_{C R}$ & $8 \times 10^{-18} \mathrm{~cm}^{3} \mathrm{~s}^{-1}$ \\
$f_{u}, f_{l}$ & $0.2916,0.0322$ & $K_{E T U 1}$ and $K_{E T U 2}$ & $1 \times 10^{-19} \mathrm{~cm}^{3} \mathrm{~s}^{-1}$ \\
$\tau_{i}(i=2,3,4)$ & $15,2.258,0.715 \mathrm{~ms}$ & $R$ & $80 \%$ \\
$\beta_{43}, \beta_{42}, \beta_{32}$ & $0.1,0.03,0.03$ & $L$ & $1.5 \%$ \\
$l, l_{1}$ & $10 \mathrm{~mm}, 5 \mathrm{~mm}$ & $A$ & $0.1256 \mathrm{~mm}^{2}$ \\
\hline
\end{tabular}

Firstly, the CW running performance of the Tm:YLF laser is numerically simulated by setting the loss $\zeta(t)$ to 0 over the entire time interval. The rate Equations (1)-(10) are solved numerically by a computer based on the four-order Runge-Kutta algorithm. As shown in Figure 6, the numerically calculated output powers increase almost linearly with incident pump powers. Under the pump power of $32 \mathrm{~W}$, the maximum average output power is $8.6 \mathrm{~W}$ for the numerical simulation, corresponding to the slope efficiency of $36.9 \%$. On the whole, the theoretical and experimental results are generally consistent, although the theoretical simulation has higher threshold power.

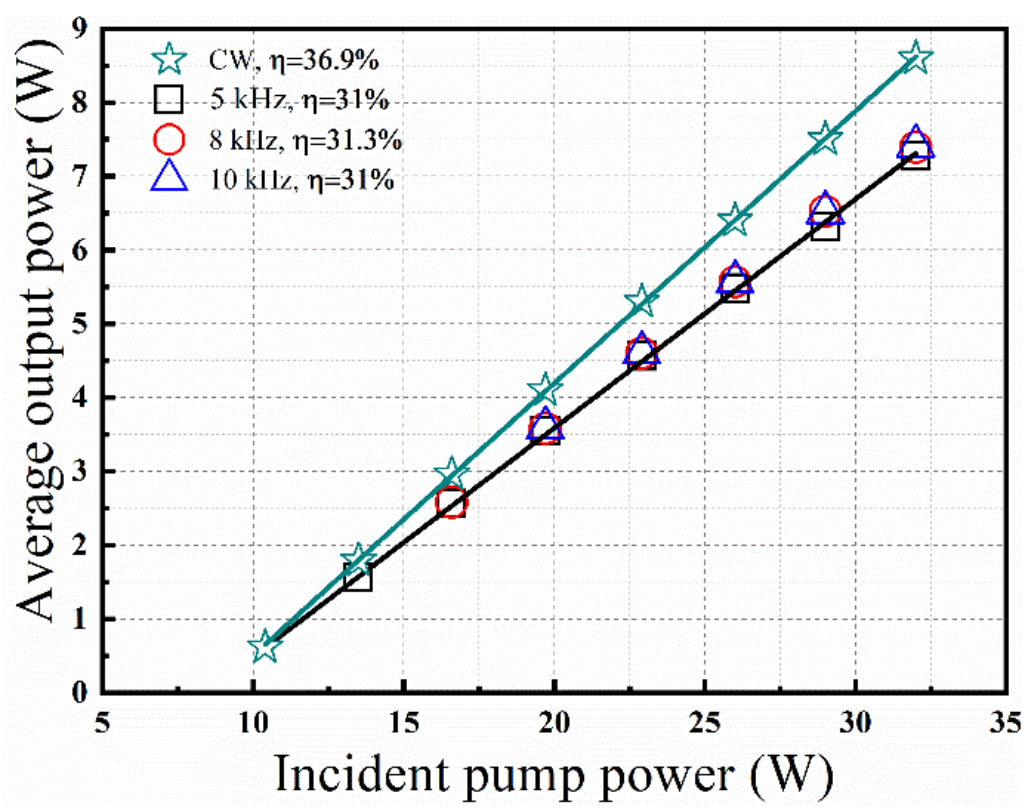

Figure 6. Numerically calculated output power versus the incident pump power for the $\mathrm{CW}$ and $\mathrm{AO}$ Q-switched Tm:YLF laser.

Then, in order to predict the pulse characteristics of the Tm:YLF laser, we assume that intracavity photon density changes to 0 instantaneously when the AO switch is turned on, and $\zeta(t)$ is 0 when the $\mathrm{AO}$ switch is off. In the numerical simulation, the maximum 
average output powers are $7.27 \mathrm{~W}, 7.39 \mathrm{~W}$ and $7.41 \mathrm{~W}$ with the slope efficiencies of $31 \%$, $31.3 \%$ and $31 \%$ at $5 \mathrm{kHz}, 8 \mathrm{kHz}$ and $10 \mathrm{kHz}$ in Figure 6 . The dependences of pulse durations, pulse energies and peak powers on incident pump powers at different repetition frequencies are recorded in Figure 7 for the numerical simulation. When the pump power is $32 \mathrm{~W}$, the experimental and simulated values are as shown in Table 2 under the repetition frequencies of $5 \mathrm{kHz}, 8 \mathrm{kHz}$, and $10 \mathrm{kHz}$. The simulated pulse energies are consistent with the experimental results, but the experimentally measured pulse widths were about 3 4 times larger than the theoretically simulated values, and the peak powers were about $3 \sim 4$ times smaller than the simulated values. Considering that the spatial distribution of the beam is ignored in the theoretical simulation, the decrease in pump density has an adverse impact on the pulse width and the peak power compared with the Gaussian transverse profile. In addition, the approximate substitution of some parameters based on the reported literature also has an adverse impact on accurate simulation.
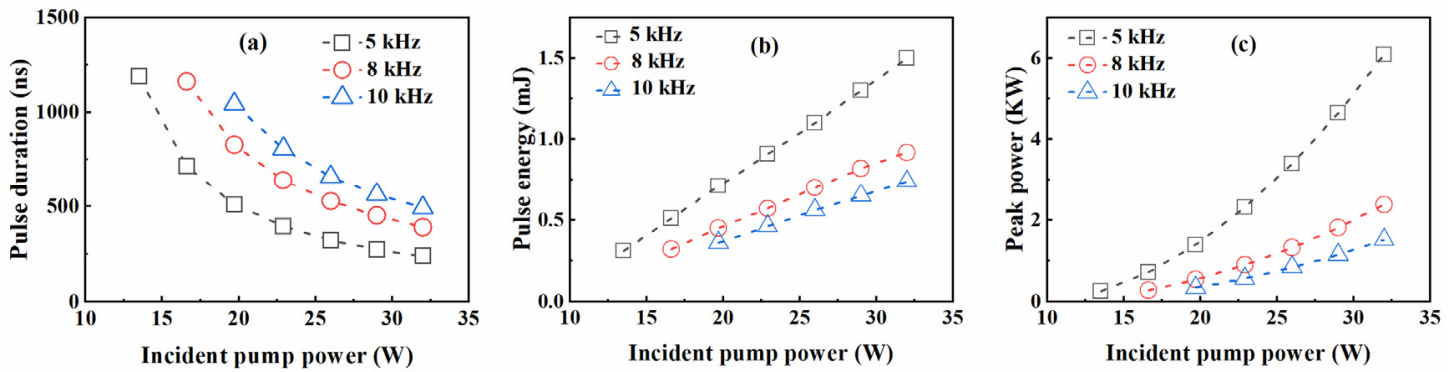

Figure 7. Simulation results for the AO Q-switched Tm:YLF laser: (a) pulse durations, (b) pulse energies and (c) peak powers versus incident pump powers.

Table 2. Comparison of experiment and simulation results (in brackets).

\begin{tabular}{cccc}
\hline $\begin{array}{c}\text { Repetition Frequencies } \\
(\mathbf{k H z})\end{array}$ & $\begin{array}{c}\text { Pulse Durations } \\
\text { (ns) }\end{array}$ & $\begin{array}{c}\text { Pulse Energies } \\
\text { (m) })\end{array}$ & $\begin{array}{c}\text { Peak Power } \\
(\mathbf{k W})\end{array}$ \\
\hline 5 & $68(239)$ & $1.46(1.45)$ & $21.5(6.1)$ \\
8 & $114(390)$ & $0.92(0.92)$ & $8.1(2.4)$ \\
10 & $140(491)$ & $0.73(0.74)$ & $5.2(1.5)$ \\
\hline
\end{tabular}

Figure 8 clearly shows the temporal evolutions of the population inversion density (gray line) and the intracavity photon density (red line) in $0.01 \mathrm{~s}$ under the maximum output power of $32 \mathrm{~W}$. As time increases, the population inversion density increases rapidly and reaches saturation near $0.002 \mathrm{~s}$. Then, the laser pulse transitions from an unstable state to a stable state, and the population inversion density also changes with the sawtooth shape. The corresponding expanded picture of the single Q-switched pulse near $0.083 \mathrm{~s}$ is shown in the inset of Figure 8. 


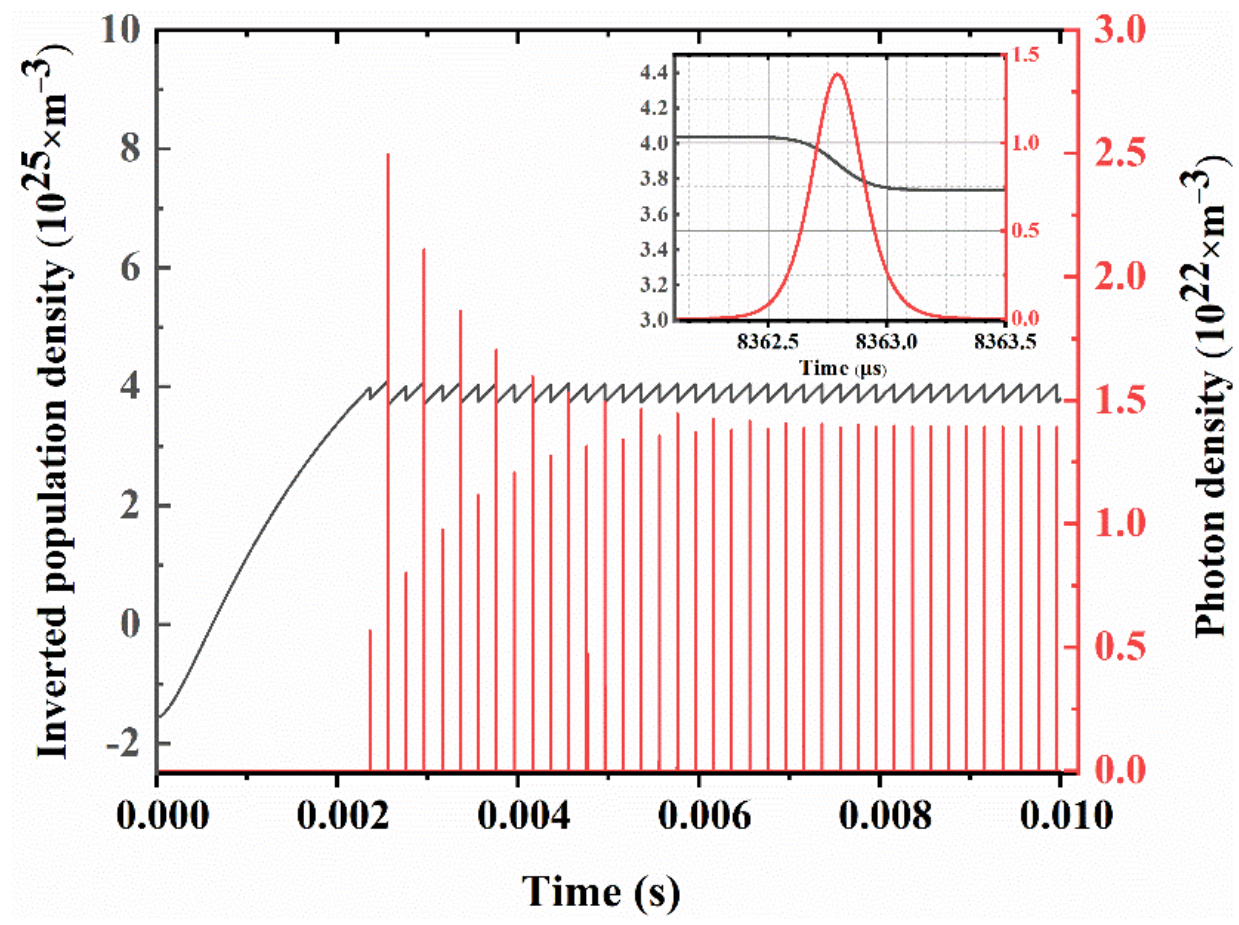

Figure 8. Population inversion density and photon density change in $0.01 \mathrm{~s}$ at the repetition frequency of $5 \mathrm{kHz}$. Inset: expanded picture of single Q-switched pulse.

\section{Conclusions}

In conclusion, a high-repetition-rate AO Q-switched Tm:YLF laser was experimentally studied at repetition rates of $5 \mathrm{kHz}, 8 \mathrm{kHz}$ and $10 \mathrm{kHz}$. Under CW operation, a maximum average output power of $8.5 \mathrm{~W}$ was obtained with a slope efficiency of $30.7 \%$. Under the $\mathrm{AO}$ Q-switching regime, a maximum output power of $7.32 \mathrm{~W}$ was obtained at the repetition rate of $5 \mathrm{kHz}$ with the shortest duration of $68 \mathrm{~ns}$ and the maximum pulse energy of $1.4 \mathrm{~mJ}$, corresponding to a maximum peak power of $21.5 \mathrm{~kW}$. In order to further analyze the experimental results, we built a quasi-three-level rate equation theoretical model of an LD-pumped AO Q-switched Tm:YLF laser, and the evolution of the pulse with time was simulated based on the four-order Runge-Kutta algorithm. However, the experimentally measured pulse widths were about $3 \sim 4$ times larger than the theoretically simulated values, which may be caused by the neglect of the Gaussian distribution of the pump and laser beams and the approximate processing of parameters in the theory. On the whole, this model can effectively predict the dependence of the pulse characteristics on the pump power, especially the average output power and the pulse energy.

Author Contributions: Conceptualization, L.G. and K.Y.; methodology, J.H.; software, L.G.; validation, Y.Y., R.W., B.Z., T.L. and S.Z.; writing-original draft preparation, L.G.; writing-review and editing, K.Y.; funding acquisition, L.G., Y.Y. and K.Y. All authors have read and agreed to the published version of the manuscript.

Funding: This research was funded by the Natural Science Foundation of Anhui Province (2108085QA29), National Natural Science Foundation of China (NSFC) (No. 62105003), University-level general projects of Anhui University of Science and Technology (xjyb2020-11), Scientific Research Foundation of Anhui University of Science and Technology, National Key Research and Development Program of China (2017YFB0405204), National Natural Science Foundation of China (NSFC) (No. 61775119), key research and development program of Shandong Province (2017CXCC0808), Shenzhen Science and Technology Research and Development Funds (JCYJ 20180305163932273) and the Program of State Key Laboratory of Quantum Optics and Quantum Optics Devices (KF201908).

Institutional Review Board Statement: Not applicable.

Informed Consent Statement: Not applicable. 
Data Availability Statement: Data are contained within this article.

Conflicts of Interest: The authors declare no conflict of interest.

Sample Availability: Samples of the compounds are available from the authors.

\section{References}

1. Li, J.; Yang, S.H.; Zhang, H.Y.; Hu, D.X.; Zhao, C.M. Diode-pumped room temperature single frequency Tm: YAP laser. Laser Phys. Lett. 2010, 7, 203-205. [CrossRef]

2. Li, G.; Yao, B.Q.; Meng, P.B.; Ju, Y.L.; Wang, Y.Z. Efficient continuous wave and Q-switched operation of a dual-end pumped c-cut Tm: YAP laser. Laser Phys. 2010, 20, 1871-1876. [CrossRef]

3. Yumoto, M.; Saito, N.; Urata, Y.; Wada, S. 128 mJ/Pulse, Laser-Diode-Pumped, Q-Switched Tm: YAG Laser. IEEE J. Sel. Top. Quant. 2014, 21, 364-368. [CrossRef]

4. Luan, C.; Yang, K.J.; Zhao, J.; Zhao, S.Z.; Qiao, W.C.; Li, T.; Feng, T.L.; Liu, C.; Qiao, J.P.; Zheng, L.H.; et al. Dual-loss-modulated Q-switched Tm:LuAG laser with AOM and monolayer grapheme. Appl. Opt. 2015, 54, 8024-8029. [CrossRef] [PubMed]

5. Shen, Y.J.; Duan, X.M.; Yuan, J.H.; Dai, T.Y.; Yao, B.Q.; Wang, Y.Z. Investigation of high-power diode-end-pumped Tm: YLF laser in slab geometry. Appl. Opt. 2015, 54, 1958-1962. [CrossRef] [PubMed]

6. Jabczyński, J.K.; Gorajek, L.; Zendzian, W.; Kwiatkowski, J.; Jelinkova, H.; Sulc, J.; Nemec, M. Efficient, high peak power, Q-switched, tunable, diode pumped Tm:YLF laser. Solid State Lasers Amplif. III 2008, 6998, 69980C.

7. Korenfeld, A.; Sebbag, D.; Ami, U.B.; Shalom, E.; Marcus, G.; Noach, S. High pulse energy passive Q-switching of a diode-pumped Tm:YLF laser by Cr:ZnSe. Laser Phys. Lett. 2015, 12, 045804. [CrossRef]

8. Sheintop, U.; Perez, E.; Sebbag, D.; Komm, P.; Marcus, G.; Noach, S. Actively Q-switched tunable narrow bandwidth milli-Joule level Tm:YLF laser. Opt. Express 2018, 26, 22135-22143. [CrossRef] [PubMed]

9. Yao, B.Q.; Meng, P.B.; Li, G.; Ju, Y.L.; Wang, Y.Z. Comparison of Tm: YLF and Tm: YAP in thermal analysis and laser performance. JOAS B 2011, 28, 1866-1873. [CrossRef]

10. Yasukevich, A.S.; Loiko, P.; Gusakova, N.V.; Serres, J.M.; Mateos, X.; Yumashev, K.V.; Kuleshov, N.V.; Petrov, V.; Griebner, U.; Aguiló, M.; et al. Modelling of graphene Q-switched Tm lasers. Opt. Commun. 2017, 389, 15-22. [CrossRef]

11. Rustad, G.; Stenersen, K. Modeling of laser-pumped Tm and Ho lasers accounting for upconversion and ground-state depletion. IEEE J. Quantum Electron. 1996, 32, 1645-1656. [CrossRef]

12. Li, G.; Liu, H.J.; Lu, F.; Wen, X.L.; Gu, Y.Q.; Wang, Y.Z. Analysis on preferential free running laser wavelength and performance modeling of $\mathrm{Tm}^{3+}$-doped YAP and YLF. Appl. Opt. 2014, 53, 4987-4996. [CrossRef] [PubMed]

13. Walsh, B.M.; Barnes, N.P.; Bartolo, B.D. Branching ratios, cross sections, and radiative lifetimes of rare earth ions in solids: Application to $\mathrm{Tm}^{3+}$ and $\mathrm{Ho}^{3+}$ ions in $\mathrm{LiYF}_{4}$. J. Appl. Phys. 1998, 83, 2772-2787. [CrossRef]

14. Xiao, Y.; Kuang, X.Y.; Yeung, Y.Y.; Ju, M. Investigation of the Structure and Luminescence Mechanism of Tm ${ }^{3+}-\mathrm{Doped} \mathrm{LiYF}_{4}$ : New Theoretical Perspectives. Inorg. Chem. 2020, 59, 1211-1217. [CrossRef] [PubMed]

15. Budni, P.A.; Lemons, M.L.; Mosto, J.R.; Chicklis, E.P. High-power/high-brightness diode-pumped 1.9- $\mu \mathrm{m}$ thulium and resonantly pumped 2.1- $\mu \mathrm{m}$ holmium lasers. IEEE J. Sel. Top. Quant. 2000, 6, 629-635. [CrossRef]

16. Evans, C.A.; Ikonic, Z.; Richards, B.; Harrison, P.; Jha, A. Theoretical modeling of a $\sim 2 \mu \mathrm{m} \mathrm{Tm}^{3+}$-doped tellurite fiber laser: The influence of cross relaxation. J. Lightwave Technol. 2009, 27, 4026-4032. [CrossRef] 